\title{
THE CASIMIR OPERATION FOR ALTERNATIVE ALGEBRAS
}

\author{
R. D. SCHAFER ${ }^{1}$
}

This note is in the nature of an addendum to our paper, Representations of alternative algebras [6]. ${ }^{2}$ The terminology and notations of that paper are used throughout. All algebras considered are finitedimensional over a field $F$ of characteristic 0 .

We generalize to alternative algebras the Casimir operation for associative algebras [3, p. 682]. However, we have been unable to obtain by this means the second Whitehead lemma for alternative algebras. This lemma is actually valid, since it is equivalent to one case of the known Wedderburn principal theorem for alternative algebras [5]. It would be desirable to have a direct proof, since then the proof of the Wedderburn principal theorem would not rely on elementwise construction for each type of split algebra. What we do prove, using the Casimir operation, is a weaker theorem (Theorem 2), which yields certain simplifications in the known proof of the Wedderburn principal theorem.

1. The Casimir operation. ${ }^{3}$ Let $\mathfrak{A}$ be an alternative algebra over $F$, and let $(S, T)$ be a representation of $\mathfrak{A}$. The $S$-kernel $\Re_{S}$ of $(S, T)$ is the set of all $x$ in $\mathfrak{A}$ such that $S_{x}=0$. Even though $x \rightarrow S_{x}$ is not generally a homomorphism, $\Omega_{S}$ is an ideal of $\mathfrak{A}$, since $x$ in $\Omega_{S}$ implies $S_{x a}=S_{x} S_{a}+\left[S_{x}, T_{a}\right]=0$ and $S_{a x}=S_{a} S_{x}-\left[S_{x}, T_{a}\right]=0$ for every $a$ in $\mathfrak{A}$ by formula $[6,(4)]$.

Now trace $S_{a b}=$ trace $S_{a} S_{b}+\operatorname{trace}\left[S_{a}, T_{b}\right]=\operatorname{trace} S_{a} S_{b}=\operatorname{trace} S_{b} S_{a}$ $=$ trace $S_{b a}$, so that

$$
\operatorname{trace} S_{a b}=\operatorname{trace} S_{b a} \quad \text { for } a, b \text { in } \mathfrak{\Re} \text {. }
$$

Also

$$
\operatorname{trace} S_{(a b) c}=\operatorname{trace} S_{a(b c)} \quad \text { for } a, b, c \text { in } \mathfrak{A}
$$

since $0=\operatorname{trace}\left[S_{b},\left[S_{a}, S_{c}\right]\right]=\operatorname{trace} S_{[b,[a, c]]-2(a, b, c)}=-2 \operatorname{trace} S_{(a, b, c)}$ by (1) and the analogue for $S$ of formula [6, (11)]. It follows from (1)

Presented to the Society, April 26, 1952; received by the editors August 24, 1952 and, in revised form, September 20, 1952.

${ }^{1}$ This research was supported in part by a contract with the Office of Naval Research.

2 Numbers in brackets refer to the references cited at the end of the paper.

${ }^{3}$ The author's knowledge of the Casimir operation and the Whitehead lemmas for Lie algebras stems not only from [1] and [3], but also from lectures on Lie algebras by C. Chevalley at Princeton University in the spring of 1948. 
and (2) that the set $\Re$ of all $x$ in $\mathfrak{A}$ satisfying

$$
\text { trace } S_{x a}=0
$$

for every $a$ in $\mathfrak{A}$

is an ideal of $\mathfrak{A}$. Clearly $\Re_{S} \leqq \Re$.

Assume now that $\mathfrak{A}$ is semisimple. Then $\Omega_{S}=\Omega$. For this is true if $\Omega=\{0\}$. If $\Omega \neq\{0\}$, then $\Omega$ is semisimple, and the unity element $e$ of $\Omega$ is an idempotent of $\mathfrak{A}$ such that $e x=x e=x$ for every $x$ in $\Omega$. Now [6, (4)] implies that $S_{e}^{2}=S_{e}{ }^{2}=S_{e}$. Hence the matrix of $S_{e}$ is similar to a matrix diag $\{I, 0\}$, and $S_{e}=0$ since trace $S_{e}=$ trace $S_{e}{ }^{2}=0$ by (3). We have $2 S_{x}=S_{e x+x e}=S_{e} S_{x}+S_{x} S_{e}=0$ for all $x$ in $\Omega$, or $\Omega \leqq \Omega s$.

Let $\mathfrak{S}_{S}$ be the (semisimple) complementary ideal of $\Omega_{S}=\Re$ in $\mathfrak{A}$. Since trace $S_{x y}$ is a nondegenerate symmetric bilinear form on $\mathfrak{S}_{S} \times \mathfrak{S}_{S}$, there is, corresponding to any basis $u_{1}, \cdots, u_{h}$ of $\mathfrak{S}_{S}$ $\left(h=\operatorname{dim} \mathfrak{S}_{S}\right)$, a dual basis $u_{1}^{*}, \cdots, u_{h}^{*}$ satisfying

$$
\text { trace } S_{u_{i} u_{j^{*}}}=\delta_{i j} \quad(i, j=1, \cdots, h)
$$

(Kronecker delta). We define the Casimir operation $\Gamma_{S}$ as the endomorphism

$$
\Gamma_{S}=\sum_{i=1}^{h} S_{u_{i}} \cdot S_{u_{i}}
$$

Theorem 1. Let $(S, T)$ be a representation of a semisimple alternative algebra $\mathfrak{A}$ of characteristic 0 , and $\Gamma_{S}$ be the Casimir operation (5). Then $\Gamma_{S}$ commutes with $S_{a}$ and $T_{a}$ for every $a$ in $\mathfrak{A}$.

Proof. Since $\mathfrak{S}_{S}$ is an ideal of $\mathfrak{A}$, we may write

$$
u_{i} a=\sum_{j=1}^{h} \alpha_{i j} u_{j}, \quad i=1, \cdots, h,
$$

for $a$ in $\mathfrak{A}$. Compute $\alpha_{k l}^{*}$ in $a u_{k}^{*}=\sum \alpha_{k j}^{*} u_{l}^{*}$ by using (4) as follows: trace $S_{\left(u_{i} a\right) u_{k^{*}}}=\sum_{j} \alpha_{i j}$ trace $S_{u_{j} u_{k^{*}}}=\alpha_{i k}$. But (2) implies that trace $S_{\left(u_{i} a\right) u_{k^{*}}}=\operatorname{trace} S_{u_{i}\left(a u_{k^{*}}\right)}=\sum_{l} \alpha_{k l}^{*}$ trace $S_{u_{i} u_{l}}=\alpha_{k l}^{*}$, so $\alpha_{i k}=\alpha_{k l}^{*}$, and

$$
a u_{i}^{*}=\sum_{j} \alpha_{j i} u_{j}^{*} \quad \text { for } \alpha_{i j} \text { in (6). }
$$

Similarly

$$
a u_{i}=\sum_{j} \alpha_{i j}^{\prime} u_{j}, \quad i=1, \cdots, h
$$

and

$$
u_{i}^{*} a=\sum_{j} \alpha_{j i}^{\prime} u_{j}^{*} \quad \text { for } \alpha_{i j}^{\prime} \text { in (8) }
$$


Then

$$
\begin{aligned}
{\left[S_{a}, \Gamma_{S}\right] } & =\sum_{i}\left(S_{a} S_{u_{i} \cdot} S_{u_{i}}-S_{u_{i} \cdot} S_{u_{i}} S_{a}\right) \\
& =\sum_{i}\left(S_{a u_{i}{ }^{*}+u_{i}{ }^{*} a} S_{u_{i}}-S_{u_{i}} S_{a u_{i}+u_{i} a}\right) \\
& =\sum_{i, j}\left(\alpha_{j i}+\alpha_{j i}^{\prime}\right) S_{u_{j} \cdot} S_{u_{i}}-\sum_{i, j}\left(\alpha_{i j}^{\prime}+\alpha_{i j}\right) S_{u_{i}} \cdot S_{u_{j}} \\
& =\sum_{i, j}\left(\alpha_{i j}+\alpha_{i j}^{\prime}\right) S_{u_{i} \cdot S_{u_{j}}}-\sum_{i, j}\left(\alpha_{i j}^{\prime}+\alpha_{i j}\right) S_{u_{i} \cdot} S_{u_{j}} \\
& =0
\end{aligned}
$$

Also

$$
\begin{aligned}
& 2\left[T_{a}, \Gamma_{S}\right]=2 \sum_{i}\left[T_{a}, S_{u_{i}} S_{u_{i}}\right] \\
& =2 \sum_{i}\left(S_{u_{i}^{*}}\left[T_{a}, S_{u_{i}}\right]+\left[T_{a}, S_{u_{i^{*}}}\right] S_{u_{i}}\right) \\
& =\sum_{i}\left(S_{u_{i} \cdot} S_{\left[a, u_{i}\right]}-S_{u_{i} \cdot}\left[S_{a}, S_{u_{i}}\right]+S_{\left[a, u_{i} \cdot\right]^{\prime}} S_{u_{i}}-\left[S_{a}, S_{u_{i} \cdot}\right] S_{u_{i}}\right) \\
& \text { by formula }[6,(27)] \\
& =\sum_{i, j}\left(\alpha_{i j}^{\prime}-\alpha_{i j}\right) S_{u_{i}:} S_{u_{j}}+\sum_{i, j}\left(\alpha_{j i}-\alpha_{j i}^{\prime}\right) S_{u_{j}:} S_{u_{i}} \\
& -\sum_{i}\left[S_{a}, S_{u_{i}}, S_{u_{i}}\right] \\
& =\sum_{i, j}\left(\alpha_{i j}^{\prime}-\alpha_{i j}\right) S_{u_{i}} S_{u_{j}}+\sum_{i, j}\left(\alpha_{i j}-\alpha_{i j}^{\prime}\right) S_{u_{i}} S_{u_{j}}-\left[S_{a}, \Gamma_{S}\right] \\
& =0 \text {. }
\end{aligned}
$$

Clearly we might have begun with the $T$-kernel of $(S, T)$ and defined a Casimir operation $\Gamma_{T}$ having the property of Theorem 1.

2. The second Whitehead lemma. Let $\mathfrak{R}$ be the radical of an alternative algebra $\mathfrak{A}$ of characteristic 0 . The Wedderburn principal theorem states that there is a subalgebra $\mathfrak{S}$ of $\mathfrak{A}$ such that $\mathfrak{S} \cong \mathfrak{A} / \mathfrak{R}$, $\mathfrak{X}=\mathfrak{S}+\mathfrak{N}$ (direct sum). ${ }^{4}$ There are two cases in the proof: (i) $\mathfrak{N}$ contains properly a nonzero ideal of $\mathfrak{A}$, (ii) $\mathfrak{N}$ does not contain properly a nonzero ideal of $\mathfrak{A}$. Case (i) may be reduced to case (ii) by an inductive argument. ${ }^{5}$

4 [5]. For arbitrary characteristic, the conclusion is valid under the assumption that $\mathfrak{A} / \mathfrak{R}$ is separable.

b In [5] the argument is referred to for the case $\mathfrak{R}^{2} \neq\{0\}$. The same proof holds for case (i). The advantage of the reduction to case (ii) instead of merely to $\mathfrak{R}^{2}=\{0\}$ is that we can then assume in Theorem 2 that $\mathfrak{B}$ is irreducible. 
Case (ii) follows readily by the method of $[3$, p. 688] from the following generalization of [3, Theorem 2.7].

Second Whitehead Lemma for alternative algebras. Let $\mathfrak{A}$ be a semisimple alternative algebra over a field $F$ of characteristic 0 , and let $\mathfrak{B}$ be an irreducible alternative module for $\mathfrak{A}$. Let $f(a, b)$ be $a$ bilinear mapping of $\mathfrak{A}$ into $\mathfrak{B}$ satisfying

$$
F(a, b, c)=F(b, c, a)=-F(b, a, c) \quad \text { for all } a, b, c \text { in } \mathfrak{A}
$$

where

$$
F(a, b, c)=f(a, b) c+f(a b, c)-a f(b, c)-f(a, b c) .
$$

Then there exists a linear mapping $g(a)$ of $\mathfrak{A}$ into $\mathfrak{B}$ such that

$$
f(a, b)=a g(b)+g(a) b-g(a b) \quad \text { for all } a, b \text { in } \mathfrak{A} \text {. }
$$

As $\mathrm{N}$. Jacobson has pointed out to us, this lemma is a consequence of-and is therefore equivalent to-(the known) case (ii). Construct an alternative algebra $\mathfrak{B}=\mathfrak{A}+\mathfrak{B}$ (direct sum) by defining multiplication in $\mathfrak{B}$ as follows:

$$
(a+v) *\left(b+v^{\prime}\right)=a b+f(a, b)+a v^{\prime}+v b
$$

for $a, b$ in $\mathfrak{A}, v, v^{\prime}$ in $\mathfrak{B}$. Since $\mathfrak{B}$ is an ideal of $\mathfrak{B}$ and $\mathfrak{B}^{2}=\{0\}$, it is sufficient to check the alternative law in $\mathscr{B}$ for products where at most one factor is in $\mathfrak{B}$. Writing

$$
[x, y, z]=(x * y) * z-x *(y * z) \quad \text { for } x, y, z \text { in } \mathfrak{B},
$$

we see that for $a, b$ in $\mathfrak{A}, v$ in $\mathfrak{B}$,

$$
[a, b, v]=(a, b, v),
$$

which alternates by $[6,(5)]$, while for $a, b, c$ in $\mathfrak{A}$

$$
[a, b, c]=(a, b, c)+F(a, b, c),
$$

which alternates by $(10)$ and the alternative law in $\mathfrak{A}$. Now $\mathfrak{B} / \mathfrak{B} \cong \mathfrak{A}$ is semisimple, so that $\mathfrak{B}$ is the radical of $\mathfrak{B}$; also $\mathfrak{B}$ contains no proper nonzero ideal of $\mathfrak{B}$ since $\mathfrak{B}$ is assumed irreducible. Case (ii) of the Wedderburn principal theorem implies that

$$
\mathfrak{B}=\mathfrak{S}+\mathfrak{B} \text { (direct sum) }
$$

where $\mathfrak{S}$ is a subalgebra of $\mathfrak{B}$. By (16) we may write any element $a$ of If uniquely in the form

$$
a=s(a)+g(a), \quad s(a) \text { in } \mathfrak{S}, g(a) \text { in } \mathfrak{B} .
$$

The mapping $a \rightarrow g(a)$ is obviously linear. Now $\subseteq$ contains $s(a) * s(b)$ 
$=\{a-g(a)\} *\{b-g(b)\}=a b+f(a, b)-a g(b)-g(a) b=s(a b)+g(a b)$ $+f(a, b)-a g(b)-g(a) b$ by (13) and (17). Since (16) is a direct sum, we have (12).

Thus the second Whitehead lemma for alternative algebras actually holds. However, we have been unable to use the Casimir operation of $\$ 1$ to give a direct proof of it. We prove instead the following weaker theorem.

Theorem 2. Let $\mathfrak{A}, \mathfrak{B}$, and $f$ be as in the second Whitehead lemma for alternative algebras. Then there exists a linear mapping $g(a)$ of $\mathfrak{A}$ into $\mathfrak{B}$ such that

$$
f^{+}(a, b)=a \cdot g(b)+g(a) \cdot b-g(a \cdot b) \quad \text { for all } a, b \text { in } \mathfrak{A}
$$

where

$$
f^{+}(a, b)=f(a, b)+f(b, a)
$$

and $a \cdot b$ and $a \cdot v$ are products in the Jordan algebra $\mathfrak{A}^{+}$and Jordan module $\mathfrak{B}^{+}$respectively $[6$, pp. 3,4$]$.

Proof. If $(S, T)$ is the zero representation $(0,0)$, we have the stronger statement (12). For the regular representation of the alternative algebra $\mathfrak{B}=\mathfrak{A}+\mathfrak{B}$ above gives a representation $\left(R^{*}, L^{*}\right)$ of $\mathfrak{A}$ in $\mathfrak{B}$. Since $\mathfrak{B} R_{a}^{*}=\mathfrak{B} L_{a}^{*}=\{0\}, \mathfrak{B}$ is invariant, and the complete reducibility of $\left(R^{*}, L^{*}\right)[6$, Theorem 2$]$ gives an invariant subspace $\subseteq$ of $\mathfrak{B}$ satisfying (16). Then we have (17), and the invariance of $\subseteq$ implies that $s(a) * b=\{a-g(a)\} * b=a b+f(a, b)=s(a b)+g(a b)+f(a, b)$ is in ऽ. Hence $f(a, b)=-g(a b)$, which is (12) for the zero representation $(0,0)$.

If $(S, T) \neq(0,0)$, we may assume that $S \neq 0$ and use the Casimir operation $\Gamma=\Gamma_{S}$. (Otherwise $T \neq 0$ and we may give a similar argument using $\Gamma_{T}$.) Hence we have $\operatorname{dim} \mathfrak{S}_{S}=h>0$, and trace $\Gamma=h>0$ by (4) and (5). In this case $\Gamma$ is nonsingular. For the subspace $\mathfrak{P}$ of all $v$ in $\mathfrak{B}$ satisfying

$$
v \Gamma=0
$$

is invariant under $(S, T)$ by Theorem 1 . Since $\mathfrak{B}$ is irreducible by hypothesis, either $\mathfrak{P}=\{0\}$ or $\mathfrak{B}=\mathfrak{B}$. In the latter case $\Gamma=0$, trace $\Gamma$ $=0$, a contradiction. Hence $\mathfrak{B}=\{0\}, \Gamma$ is nonsingular.

Write $h(a)=\sum_{i} f\left(a, u_{i}^{*}\right) S_{u_{i}}$. Since

$$
\begin{aligned}
\sum_{i} f\left(b, a u_{i}^{*}\right) S_{u_{i}} & =\sum_{i, j} \alpha_{j i} f\left(b, u_{j}^{*}\right) S_{u_{i}} \\
& =\sum_{i, j} \alpha_{i j} f\left(b, u_{i}^{*}\right) S_{u_{j}}=\sum_{i} f\left(b, u_{i}^{*}\right) S_{u_{i} a}
\end{aligned}
$$


by (6) and (7), we have

$$
\begin{aligned}
& f(b, a) \Gamma-h(b) a-b h(a)+h(b a) \\
& =\sum_{i}\left\{f(b, a) S_{u_{i}} S_{u_{i}}-f\left(b, u_{i}^{*}\right) S_{u_{i}} S_{a}-f\left(a, u_{i}^{*}\right) S_{u_{i}} T_{b}+f\left(b a, u_{i}^{*}\right) S_{u_{i}}\right\} \\
& =\sum_{i}\left\{F\left(b, a, u_{i}^{*}\right) S_{u_{i}}+f\left(a, u_{i}^{*}\right)\left[T_{b}, S_{u_{i}}\right]\right. \\
& \left.+f\left(b, a u_{i}^{*}\right) S_{u_{i}}-f\left(b, u_{i}^{*}\right) S_{u_{i}} S_{a}\right\} \\
& =\sum_{i}\left\{F\left(b, a, u_{i}^{*}\right) S_{u_{i}}+f\left(a, u_{i}^{*}\right)\left(S_{u_{i}} S_{b}-S_{u_{i} b}\right)\right. \\
& \left.\left.+f\left(b, u_{i}^{*}\right)\left(S_{u_{i} a}-S_{u_{i}} S_{a}\right)\right\} \quad \text { (by }[6,(4)]\right) \text {. }
\end{aligned}
$$

Interchange $a$ and $b$, and add, to obtain

$$
f^{+}(a, b) \Gamma-a \cdot h(b)-h(a) \cdot b+h(a \cdot b)=0
$$

by (10). Then (21) implies (18) for $g(a)=h(a) \Gamma^{-1}$ by Theorem 1 .

In case (ii) of the Wedderburn principal theorem, Theorem 2 has the force of the Wedderburn principal theorem for $\mathfrak{A}^{+}$: there is a (Jordan) subalgebra $\mathfrak{S}^{+}$of $\mathfrak{Q}^{+}$such that $\mathfrak{S}^{+} \cong \mathfrak{U}^{+} / \mathfrak{N}^{+}, \mathfrak{A}^{+}=\mathfrak{S}^{+}+\mathfrak{N}^{+}$ (direct sum). Equivalently, there is a subspace $\mathfrak{S}$ of $\mathfrak{A}$, closed with respect to the Jordan product $a \cdot b$, such that $\mathfrak{S}^{+} \cong(\mathfrak{A} / \mathfrak{N})^{+}, \mathfrak{R}=\mathfrak{S}+\mathfrak{N}$ (direct sum). This follows by the method of [3, p. 688].

For if $\mathfrak{A}=\mathfrak{T}+\mathfrak{N}$ (direct sum) where $\mathfrak{T}$ is a subspace of $\mathfrak{A}$, then there exists a linear isomorphism $a \rightarrow a^{*}$ between the spaces $\mathfrak{R} / \mathfrak{l}$ and $\mathfrak{I}\left(a\right.$ in $\mathfrak{A} / \mathfrak{N}, a^{*}$ in $\left.\mathfrak{T}\right)$ such that $(a b)^{*}-a^{*} b^{*}$ is in $\mathfrak{R}$. Write

$$
f(a, b)=(a b)^{*}-a^{*} b^{*}
$$

in $\mathfrak{N}$. Since $\mathfrak{N}^{2}=\{0\}, \mathfrak{N}$ is an alternative module for $\mathfrak{A} / \mathfrak{N}$ when we define

$$
v a=v S_{a}=v a^{*}, \quad a v=v T_{a}=a^{*} v \quad \text { for } v \text { in } \mathfrak{N}, a \text { in } \mathfrak{Y} / \mathfrak{N} .
$$

Also $f(a, b)$ is a bilinear mapping of $\mathfrak{A} / \mathfrak{N}$ into $\mathfrak{N}$ satisfying (11). For compute $F(a, b, c)=(a b)^{*} c^{*}-\left(a^{*} b^{*}\right) c^{*}+\{(a b) c\}^{*}-(a b)^{*} c^{*}-a^{*}(b c)^{*}$ $+a^{*}\left(b^{*} c^{*}\right)-\{a(b c)\}^{*}+a^{*}(b c)^{*}=(a, b, c)^{*}-\left(a^{*}, b^{*}, c^{*}\right)$. Then (10) follows from the alternative law in $\mathfrak{A}$. Since we are assuming that $\mathfrak{R}$ does not contain properly any nonzero ideal of $\mathfrak{A}$, the alternative module $\mathfrak{N}$ is irreducible. Theorem 2 guarantees the existence of a linear mapping $a \rightarrow g(a)$ of $\mathfrak{A} / \mathfrak{N}$ into $\mathfrak{N}$ satisfying (18); that is,

$$
(a \cdot b)^{*}-a^{*} \cdot b^{*}=a^{*} \cdot g(b)+g(a) \cdot b^{*}-g(a \cdot b) .
$$

Setting $a^{\prime}=a^{*}+g(a)$ in $\mathfrak{A}$, we have $a^{\prime} \cdot b^{\prime}=a^{*} \cdot b^{*}+a^{*} \cdot g(b)+g(a) \cdot b^{*}$ $=(a \cdot b)^{*}+g(a \cdot b)=(a \cdot b)^{\prime}$, so that $a \rightarrow a^{\prime}$ is an isomorphism of $(\mathfrak{A} / \mathfrak{R})^{+}$ into $\mathfrak{U}^{+}$. 
3. The Wedderburn principal theorem. We refer to our proof of the Wedderburn principal theorem for alternative algebras [5]. We show, in particular, how the proofs of Lemmas 3 and 5 may be simplified by the result of the previous section. ${ }^{6}$

We first remark that elements $e_{i}$ are pairwise orthogonal idempotents (p.o.i.) in $\mathfrak{A}$ if and only if the elements $e_{i} / 2$ are p.o.i. in $\mathfrak{A}^{+}$. This is obvious except to note that, if $e_{i} \cdot e_{j}=0$ for idempotents $e_{i}$ and $e_{j}$ in $\mathfrak{A}$, then $e_{i} e_{j}=0$. By the Theorem of Artin, $e_{i} e_{j}+e_{j} e_{i}=0 \mathrm{im}$ plies $0=e_{i} e_{j}+e_{i} e_{j} e_{i}=e_{i} e_{j} e_{i}+e_{j} e_{i}$; adding, $2 e_{i} e_{j} e_{i}=0$, or $e_{i} e_{j}=0$.

Then, if $\left[u_{1}\right], \cdots,\left[u_{s}\right]$ are p.o.i. in $\mathfrak{A} / \mathfrak{N}$, there exist p.o.i. $e_{1}, \cdots, e_{s}$ in $\Re$ such that $\left[e_{i}\right]=\left[u_{i}\right][5$, Lemma 3]. For the elements $\left[u_{i}\right] / 2$ are p.o.i. in $(\mathfrak{A} / \mathfrak{N})^{+}$. By $\$ 2$ there exist p.o.i. $e_{i} / 2$ in $\mathfrak{I}^{+}$such that $\left[e_{i} / 2\right]=\left[u_{i}\right] / 2$, and the elements $e_{i}$ are p.o.i. in $\mathfrak{A}$ as desired.

We use the result of $\$ 2$ to simplify the proof of [5, Lemma 5] as follows: given that $\mathfrak{A} / \mathfrak{N}$ is the Cayley-Dickson algebra $\mathbb{C}=\mathfrak{M}_{2}$ $+[w] \mathfrak{M}_{2}$ with divisors of zero, we are to prove that $\mathfrak{A}=\mathfrak{G}_{1}+\mathfrak{R}$, $\mathfrak{G}_{1} \cong \mathfrak{E}$. We are concerned only with the case where $\mathfrak{A}$ has a unity element $e$, and where $\mathfrak{N}^{2}=0$. By $\S 2$ we know that $\mathfrak{U}=\mathfrak{S}+\mathfrak{N}$ where $\mathfrak{S}^{+} \cong \mathfrak{S}^{+}$. There exist orthogonal idempotents $e_{11}, e_{22}$ in $\mathfrak{S}$ such that $e=e_{11}+e_{22}$ and the elements $\left[e_{i i}\right]$ are contained in the matric basis of $\mathfrak{M}_{2}$. Let $s$ be the element of $\mathfrak{S}$ such that $[s]=\left[e_{12}\right]+\left[e_{21}\right]$ in $\mathfrak{M}_{2}$. Then $\mathfrak{S}^{+} \cong \mathfrak{S}^{+}$implies $s \cdot s=2 e, e_{i i} \cdot s=s$, so that

$$
s^{2}=e, \quad e_{i i} s=s e_{j j} \quad(i \neq j) .
$$

Write $e_{i j}=e_{i i} s(i \neq j)$. It follows easily from (25) that the elements $e_{11}, e_{12}, e_{21}, e_{22}$ are a matric basis of a total matric subalgebra $\mathfrak{M}$ of $\mathfrak{A}$. $\mathfrak{M}_{2}$ is the image of $\mathfrak{M}$ under the natural homomorphism of $\mathfrak{A}$ onto $\mathfrak{E}=\mathfrak{M}_{2}+[w] \mathfrak{M}_{2}$. In order to prove $[5$, Lemma 5$]$, it is sufficient to show the existence of a pair $p_{12}, p_{21}$ of elements $p_{i j}$ in $\mathfrak{A}_{i j}$ satisfying

$$
p_{i j} p_{j i}=e_{i i}, \quad e_{i j} p_{j i}=p_{j i} e_{i j}=0 \quad(i \neq j) .
$$

Choose the representative $w$ of $[w]$ in $\subseteq$. Then $w \cdot w=2 e, s \cdot w=0$, $e_{i i} \cdot w=w$ imply

$$
w^{2}=e, \quad s w=-w s, \quad e_{i i} w=w e_{j j} \quad(i \neq j) .
$$

For $i \neq j$, write

$$
f_{i j}=e_{i i} w=w e_{j j} \quad \text { in } \mathfrak{A}_{i j} .
$$

Then

- Of course the same effect is achieved by assuming the Wedderburn principal theorem for $\mathfrak{A}^{+}[\mathbf{4}]$. Note that the only simple Jordan algebras encountered in $(\mathfrak{A} / \mathfrak{R})^{+}$ $=\mathfrak{U}^{+} / \mathfrak{R}^{+}$are of types $\mathrm{A}$ and $\mathrm{D}$. 


$$
f_{j i} e_{i j}=-e_{j i} f_{i j}
$$

in $\mathfrak{N} \cap \mathfrak{U}_{j \boldsymbol{j}}$,

since $\quad f_{j i} e_{i j}=\left(e_{j j} w\right)\left(s e_{j j}\right)=e_{j j}(w s) e_{j j}=-e_{j j}(s w) e_{j j}=-\left(e_{j j} s\right)\left(w e_{j j}\right)$ $=-e_{j i} f_{i j}$ by (27) and the Moufang lemma, while $\left[f_{j i} e_{i j}\right]=\left([w]\left[e_{i i}\right]\right)\left[e_{i j}\right]$ $=[w]\left(\left[e_{i j} e_{i i}\right]\right)=[0]$. Using (28), we write

$$
p_{i j}=f_{i j}+e_{i j}\left(f_{j i} e_{i j}\right)=f_{i j}-e_{i j}\left(e_{j i} f_{i j}\right) .
$$

Then

$$
\begin{aligned}
p_{i j} p_{j i} & =f_{i j} f_{j i}+f_{i j}\left\{e_{j i}\left(f_{i j} e_{j i}\right)\right\}+\left\{e_{i j}\left(f_{j i} e_{i j}\right)\right\} f_{j i}=\left(e_{i i} w\right)\left(w e_{i i}\right)+2\left(f_{i j} e_{j i}\right)^{2} \\
& =e_{i i} .
\end{aligned}
$$

Also $e_{i j} p_{j i}=e_{i j} f_{j i}-e_{i j}\left\{e_{j i}\left(e_{i j} f_{j i}\right)\right\}=e_{i j} f_{j i}-\left(e_{i j} e_{j i}\right)\left(e_{i j} f_{j i}\right)=0$ by [5, (22)], and similarly $p_{j i} e_{i j}=0$. The elements $p_{i j}$ in (29) satisfy (26), as desired.

\section{REFERENCES}

1. C. Chevalley and S. Eilenberg, Cohomology theory of Lie groups and Lie algebras, Trans. Amer. Math. Soc. vol. 63 (1948) pp. 85-124.

2. S. Eilenberg, Extensions of general algebras, Annales de la Société Polonaise de Mathematique vol. 21 (1948) pp. 125-134.

3. G. Hochschild, Semisimple algebras and generalized derivations, Amer.' J. Math. vol. 64 (1942) pp. 677-694.

4. A. J. Penico, The Wedderburn principal theorem for Jordan algebras, Trans. Amer. Math. Soc. vol. 70 (1951) pp. 404-420.

5. R. D. Schafer, The Wedderburn principal theorem for alternative algebras, Bull. Amer. Math. Soc. vol. 55 (1949) pp. 604-614.

6. - Representations of alternative algebras, Trans. Amer. Math. Soc. vol. 72 (1952) pp. 1-17.

University of Pennsylvania 